\title{
Glia maturation factor-y (GMFG) promotes ovarian tumorigenesis and chemoresistance via activation of the FAK signaling-associated focal adhesion dynamics
}

\author{
Aihua Lan \\ the Fifth People's Hospital of Shanghai, Fudan University \\ Cuiting LV \\ the Fifth People's Hospital of Shanghai, Fudan University \\ Jingshu Wang \\ the Fifth People's Hospital of Shanghai, Fudan University \\ Lina Yang \\ the Fifth People's Hospital of Shanghai, Fudan University

\section{Ran Du} \\ the Fifth People's Hospital of Shanghai, Fudan University \\ Hui Li \\ the Fifth People's Hospital of Shanghai, Fudan University \\ Yinjue Yu \\ the Fifth People's Hospital of Shanghai, Fudan University

\section{Chunxia Ren} \\ Shuguang Hospital, Shanghai University of Traditional Chinese Medicine \\ Gong Yang ( $\nabla$ yanggong@fudan.edu.cn ) \\ the Fifth People's Hospital of Shanghai, Fudan University
}

\section{Research Article}

Keywords: Ovarian cancer, Glia maturation factor-y, Focal adhesion kinase, Extracellular matrix protein 1, Chemoresistance

Posted Date: May 19th, 2022

DOI: https://doi.org/10.21203/rs.3.rs-801035/v2

License: (c) (1) This work is licensed under a Creative Commons Attribution 4.0 International License.

Read Full License 


\section{Abstract}

Glia maturation factor- $($ GMFG) is reported to regulate actin cytoskeleton remodeling through the facilitation of actin debranching and nucleation suppression, which may be associated with cellular malignancy, but the role of GMFG in tumorigenesis remains largely unknown. By overexpressing or silencing of GMFG in ovarian cancer cell lines, we show that GMFG enhances in vitro ovarian cancer cell proliferation, migration, invasion, and paclitaxel resistance and accelerates in vivo tumor growth and intraperitoneal metastasis in xenograft animal models. The mechanistic study demonstrates that GMFG activates the FAK/Talin/Paxillin/Src signaling molecules via binding to p-FAK (Tyr397) and p-Talin (Ser425), whereas cell proliferation, migration and paclitaxel resistance induced by GMFG can be inversely suppressed by the chemical inhibition of p-FAK (Tyr397). Additionally, patients with high expression of GMFG exhibited a poor progression-free survival (PFS) (HR =1.42, 95\%Cl: $1.1-1.82, \mathrm{P}=$ 0.0065). To conclude, our study suggests that GMFG may activate FAK signaling via binding to $p$-FAK (tyr397) and p-Talin (ser425) to promote ovarian tumorigenesis and chemoresistance. These findings indicate a functional interaction between GMFG and FAK pathway in ovarian tumorigenesis and chemoresistance. Thus, targeting the oncogenic GMFG-FAK axis may be a promising therapeutic strategy for ovarian cancer.

\section{Introduction}

Ovarian cancer consists of $2.5 \%$ of all malignancies for women, but for $5 \%$ of all cancer-associated deaths because of its high mortality ${ }^{1}$. Up to $80 \%$ of ovarian cancer patients are diagnosed at advanced stages due to lack of early symptoms, the 5-year survival rates of these patients diagnosed at localized, regional and distant stages are around $90 \%, 70 \%$ and $30 \%$, respectively ${ }^{2,3}$. In order to inhibit the progression of ovarian cancer and to improve the prognosis of patients, investigation on the ovarian tumorigenic mechanism is clinically demanded.

Our previous study found that Extracellular matrix protein 1a (ECM1a) enhances the progression of ovarian cancer through activation of the Focal adhesion kinase (FAK) signaling ${ }^{4}$, and by RNA-seq analysis, we further revealed that Glia maturation factor- $\mathrm{Y}$ (GMFG) was significantly downregulated in ECM1-knockdown ovarian cancer cells, but was significantly upregulated in ECM1a-overexpression cells. Moreover, through analyzing patients' survival data from The Cancer Genome Atlas (TCGA) database, we found that GMFG is a prognostic factor for several cancer types ${ }^{5}$. GMFG belongs to the actindepolymerizing factor homology (ADF-H) family and functions in actin reorganization through interacting with actin-related proteins (Arp) 2/3 complex to promote the motility of several cell types, including neutrophils and T lymphocytes 6,7 . Thus, GMFG may have a great potential to induce tumorigenicity in terms of the actin remodeling. Focal adhesions (FAs) are large multi-protein complexes including FAK, Talin, Paxillin and Src, which are localized between the Extracellular matrix (ECM) and the cytoskeleton. FAs attach cells to ECM via the transmembrane integrins to transduce the signals associated with cell adhesion, proliferation and survival ${ }^{8,9}$. The phosphorylation of FAK at Tyr397 is vital for the FAK 
signaling activation and FAs turnover, and is thereby critical for the motility, survival and proliferation of adhesion-dependent cells ${ }^{10}$. It has been well-studied that FAK is overexpressed and/or overphosphorylated in various cancer types, leading to cell migration ${ }^{11,12}$, proliferation ${ }^{13}$, and metastasis ${ }^{14}$.

Considering the close interaction between cytoskeleton remodeling and FAs dynamics, this study was aimed to investigate the function of GMFG in ovarian cancer cells, the impact of GMFG on FAK pathway, and the relationship between GMFG and ECM1 in terms of the FAK signaling modulation. By conducting in vitro functional assays, in vivo xenograft tumor formation experiments, and analysis of the associated signal molecules, we demonstrate that GMFG may link ECM1 and FAK to promote the progression of ovarian cancer via the FAK/Talin/Paxillin/Src-induced FAs dynamics.

\section{Materials And Methods}

\section{Cell lines and cell culture}

All cell lines used in this study were listed using the official cell line name and its Research Resource Identifier (RRID) as available in the ExPASy Cellosaurus database (https://web.expasy.org/cellosaurus/). Human ovarian epithelial cancer cell lines HEY (RRID:CVCL_0297), HEY A8 (RRID:CVCL_8878, the isogenic cell lines of HEY) ${ }^{15}$, SK-OV-3 (RRID:CVCL_0532), A2780 (RRID:CVCL_0134), OVCA433 (RRID:CVCL_0475), OVCA429 (RRID:CVCL_3936) and lentivirus packaging cell line HEK293T (RRID:CVCL_0063) were obtained from the American Tissue Culture Collection (ATCC), immortalized normal human ovarian surface epithelial cell line T29 (RRID:CVCL_2G67) ${ }^{4}$, and normal human ovarian

surface epithelial cell line NOE (also called HOSE) were established in our lab ${ }^{16}$. All human cell lines have been authenticated using STR profiling by Genetic Testing Biotechnology (Suzhou, China) within the last three years. All experiments were performed with mycoplasma-free cells. All cell lines were cultured using Roswell Park Memorial Institute (RPMI) 1640 in a humidified incubator with an atmosphere of $5 \% \mathrm{CO}_{2}$ at $37^{\circ} \mathrm{C}$, with the exception of $293 \mathrm{~T}$ which was maintained in Dulbecco's modified Eagle's medium (DMEM). $10 \%$ fetal bovine serum (FBS), $2 \mathrm{mM} \mathrm{L-glutamine,} \mathrm{penicillin} \mathrm{(100} \mathrm{units} / \mathrm{ml}$ ) and streptomycin $(100 \mu \mathrm{g} / \mathrm{ml})$ were added to the culture media.

\section{Stable cell lines construction}

To knockdown GMFG and ECM1, synthesized DNA oligos were designed to generate small hairpin RNA (shRNAs) targeting GMFG-specific mRNAs and ECM1-specific mRNAs, the specific shRNAs and a scrambled shRNA (negative control) were inserted into plko.1/puromycin vectors separately. To overexpress GMFG and ECM1a, the CDNA of GMFG and ECM1a were cloned and inserted into PCDHneomycin vectors along with a Flag-tag and a HA-tag respectively, and an empty PCDH-neomycin vector was used to generate control cell lines. All related primers were listed in Supplementary Table 1, plasmids and stable cell lines were constructed following previous research pipeline ${ }^{4}$.

\section{Quantitative real-time polymerase chain reaction (qPCR)}


To perform qPCR, total RNA was extracted from cells and isolated with TRIzol reagent (Invitrogen) according to the manufacturer's instructions, and RNA reverse transcription was conducted to generate cDNA using a PrimeScript ${ }^{\text {TM }}$ RT Master Mix (TaKaRa) following the manufacturer's instructions. To test the mRNA expression levels of specific genes, qPCR was carried out on the Applied Biosystems 7500 Real-Time PCR Systems (Applied Biosystems) using FastStart Universal SYBR Green Master (ROX) kit (Roche) with the generated cDNA and specific qPCR primers. GAPDH was applied as the internal standard, and all qPCR primers used in this study were showed in Supplementary Table 1. The relevant mRNA expression values were measured using the $\Delta \Delta \mathrm{C}_{\mathrm{t}}$ method.

\section{Western blot (WB) analysis}

Protein samples were collected from cell lysates using RIPA buffer added with a protease inhibitor cocktail, and then the expression levels of different proteins were detected by standard WB procedures ${ }^{4}$. The detailed information of the primary antibodies applied in the present study was summarized in Supplementary Table 2. The secondary antibodies against mouse (\#7076) or rabbit lgG (\#7074) were purchased from Cell Signaling Technology (Massachusetts, USA). The immunoblots in our study were visualized using Fluorchem $\mathrm{E}$ (ProteinSimple, USA) and enhanced chemiluminescence reagent (Thermo Scientific, USA).

\section{Co-Immunoprecipitation (Co-IP)}

Co-IP assay was conducted according to the standard protocol using protein G-agarose (Roche). After several washes in ice-cold PBS, the crude extract of cells was suspended using ice-cold lysis buffer at a recommended concentration. $25 \mu \mathrm{L}$ protein G-agarose was used to decrease the background for no less than 30 minutes at $4^{\circ} \mathrm{C}$, then a specific antibody at a recommended amount was added to the cell supernatant for incubation overnight at $4^{\circ} \mathrm{C}$, and the same amount of a normal lgG of the same species was used as the negative control. After antibody incubation, $50 \mu \mathrm{L}$ protein $\mathrm{G}$-agarose was used to form agarose-antibody-antigen complex, and the mixture was washed using the recommended washing buffer for several times. Finally, bound proteins were detected by routine WB analysis. The antibodies used in Co-IP assay were listed in Supplementary Table 2, and normal IgG applied in Co-IP assay was either rabbit IgG (SC\#2027, Santa Cruz Biotech, CA, USA) or mouse IgG (SC\#2025, Santa Cruz Biotech, CA, USA).

\section{Immunofluorescence (IF) staining}

IF staining was performed using published method ${ }^{17}$. Briefly, an appropriate density of cells was seeded and incubated overnight in 24-well plates with cover slides. The cover slides were washed using PBS and fixed by precooled methanol for 15 minutes. $0.5 \%$ Triton X-100 was used to permeabilize the cells for 5 min, then cells were blocked by $5 \%$ BSA. All cell samples were treated with double staining in this study, cells were incubated with a mixture of two different primary antibodies at an appropriate concentration and then secondary antibodies under dark condition. Finally, cells were washed again with PBS and stained with DAPI. The information of applied primary antibodies was showed in Supplementary Table 2. 
The secondary antibodies used in IF staining were Alexa Fluor 594 AffiniPure donkey anti-rabbit IgG (green) and Fluor 594 AffiniPure donkey anti-mouse IgG (red). All stained cells were observed using a fluorescence microscope and photographed by a fluorescent microscope camera.

\section{Cell migration and invasion assay}

Wound healing assay was carried out to detect cell migration speed. Cells were placed and incubated in six-well plate overnight. A sterile P200 pipette tip was used to form a scratch, and warm PBS was used to wash the floating cells away. The migration speed was recorded by taking photographs at 0 hour and 24 hours.

Besides, cell migration and invasion abilities were also measured using the transwell chamber assay. For migration assay, an appropriate number of cancer cells in $100 \mu \mathrm{L} \mathrm{FBS-free} \mathrm{RPMI} 1640$ was seeded directly into the upper chambers, and about $600 \mu \mathrm{L}$ RPMI 1640 supplemented with $10 \%$ FBS was added into the lower chambers. For invasion assay, the upper chambers were firstly added with Matrigel (BD Biosciences) following the manufacturer's protocol and the cells were then placed on top of the Matrigel in the upper chamber. After culturing for about 24 hours in incubator, the invaded cells were fixed by methanol, stained by $0.1 \%$ crystal violet for about 30 minutes, and the images were captured by a light microscope. Experiments were performed in triplicate.

\section{Plate colony formation assay}

500 cells were seeded in six-well plates at a single-cell density. Fresh RPMI 1640 containing 10\% FBS was added every 3-5 days to allow cell growth until visible cell colonies could be observed by unaided eye. Then colonies were fixed using methanol, stained by $0.1 \%$ crystal violet, manually counted and analyzed.

\section{Soft agar anchorage-independent growth assays}

$2.5 \mathrm{ml}$ RPMl 1640 containing 20\% FBS and $2.5 \mathrm{ml} \mathrm{1.5 \%} \mathrm{agarose} \mathrm{(Sigma-Aldrich,} \mathrm{United} \mathrm{States)} \mathrm{were}$ added to $6 \mathrm{~cm}^{2}$ plate as the lower agar. Then 5,000 cancer cells in $0.5 \mathrm{ml} \mathrm{RPMl} 1640$ medium containing $20 \%$ FBS with $0.5 \mathrm{ml} 0.7 \%$ agar were mixed and placed onto the lower agar. After incubation for about 3 weeks, the colonies were photographed and counted under microscope. Colony formation assays were performed in triplicate.

\section{Cell proliferation assay}

A total of $1 \times 10^{3}$ cells in $100 \mu$ L RPMI 1640 containing $10 \%$ FBS were placed in 96-well culture plates. Cell proliferation capacity was detected at the $1,2,3$, and 4 days by the cell counting kit-8 (CCK-8) (Dojindo, Tokyo, Japan) following the manufacturer's instruction at $450 \mathrm{~nm}$ (Tecan Infinity 200PRO multiwell plate reader). The assays were independently conducted three times. 
$1 \times 10^{4}$ cells in $100 \mu \mathrm{L}$ RPMI 1640 per well were seeded in 96-well culture plates and incubated overnight, then fresh RPMI 1640 containing different concentrations of paclitaxel (Haosen pharmaceutical company, Jiangsu, China) was used to culture cells. After culturing with paclitaxel for 48 hours, cell viability was detected by CCK-8 method described above, and IC50 value was calculated.

To inhibit the phosphorylation of FAK at the Tyr397 site, three different specific inhibitors (Defactinib hydrochloride, MedChemExpress, HY-12289A; GSK2256098, Selleckchem; and PF573228, Selleckchem) were applied to HEY-G cells for 8,12 and 24 hours respectively. Then inhibitor-treated HEY-G cells, DMSOtreated HEY-G cells (diluent, the same volume of DMSO was used as negative control) and blank HEY-G cells (no treatment) were collected for WB analysis to confirm that FAK signaling pathway was inhibited. After that, Defactinib-treated, DMSO-treated, and blank HEY-G cells were used to conduct plate colony formation experiment, cell proliferation assay and wound healing assay according to the methods described previously. The concentrations of Defactinib and DMSO were maintained during the experimental period.

\section{Xenograft tumor model establishment}

The animal assays approved by the Institutional Animal Care and Use Committee of East China Normal University (reference number: m20200801) were carried out in accordance with the ARRIVE guidelines. 6week-old female BALB/c nude mice from Slac Laboratory Animal (Shanghai, China) were subjected to intraperitoneal injection of $1 \times 10^{7}$ cells to establish xenograft tumor models. mice were observed regularly and killed before natural death occurred, then the mice were weighed and tumor nodules were taken out, counted, weighed and calculated.

\section{Immunohistochemistry (IHC)}

Murine tumor tissues were fixed in $10 \%$ formalin and embedded in paraffin, later were cut into $5-\mu m$ thick. Tissue sections were stained according to our previously published method ${ }^{4}$. The relevant information of the primary antibodies used to stain tissues were summarized in Supplementary Table 2, and the secondary antibodies were from EnVision ${ }^{\text {TM }}$ Detection Kit (GENE, USA). The images were captured at 200x magnification.

\section{Kaplan-Meier analysis}

Kaplan-Meier plot was generated by Kaplan-Meier Plotter website (http://www.kmplot.com) ${ }^{18}$ to assess the prognostic values of GMFG in ovarian cancer, hazard ratio (HR) with $95 \%$ confidence interval $(95 \% \mathrm{Cl})$ and log-rank $P$ value were calculated. Patients were collected from TCGA database and were divided into high and low GMFG expression groups according to the best cutoff value.

\section{Statistical analysis}

All data were analyzed by an unpaired two-tailed Student's t test, and statistical significance was set at ${ }^{*} p \leq 0.05, * \star p \leq 0.01, * \star * p \leq 0.001$. 


\section{Results}

\section{ECM1-induced GMFG activates the FAK-associated signaling pathway}

We previously investigated the tumorigenic function of ECM1 in ovarian cancer ${ }^{4}$, in which we first silenced ECM1 in wild-type ovarian cancer HEY A8 cell line (HEY A8-ECM1i, subsequently abbreviated "HEY A8-Ei", where "i" refers to interfering RNA as in other labels), and then we overexpressed the subtype ECM1a in HEY A8-Ei cells (HEY A8-Ei-ECM1a, subsequently abbreviated "HEY A8-Ei-A") (Figure 1A). We found that silencing of ECM1 downregulated the expression levels of FAK, p-FAK (Tyr397), Talin, pTalin (Ser425), Paxillin, p-Paxillin (Tyr118), Src and p-Src (Tyr416), but overexpression of ECM1a upregulated the expression of these proteins, compared with their relative controls (Figure 1B). RNA-seq analysis revealed that GMFG was downregulated in HEY A8-Ei, but was upregulated in HEY A8-Ei-A (Supplementary Table 3), which was confirmed by the examination of both GMFG mRNA and protein levels in HEY A8, HEY A8-Ei and HEY A8-Ei-A cells by qRT-PCR and WB shown in Figure 1C-D.

To investigate the function of GMFG in ovarian cancer, we first evaluated the expression levels of GMFG by qRT-PCR and WB in six human ovarian cancer cell lines (HEY A8, HEY, SKOV3, A2780, OVCA429 and OVCA433), and two normal human ovarian surface epithelial cell lines (T29 and NOE). We found that GMFG was higher in HEY A8 and SKOV3 cell lines than in HEY and OVCA433 cell lines (Figure 1E-F). Thus, HEY A8 and SKOV3 were chosen to establish GMFG knockdown cell lines by the shRNA-mediated stable gene silencing, whereas HEY and OVCA433 were selected to generate GMFG overexpression cell lines by introduction of exogenous GMFG CDNA in these cell lines. As tested by qRT-PCR and WB, the expression of GMFG was markedly increased in both HEY-GMFG (HEY-G) and OVCA433-GMFG (OVCA433-G) cells compared with in their corresponding vector control cells (HEY-V and OVCA433-V), and the tagged GMFG with Flag was also tested by antibody to Flag in HEY-G and OVCA433-G (Figure 1G-H). In contrast, GMFG was decreased in HEY A8-Gi-1, HEY A8-Gi-2, SKOV3-Gi-1 and SKOV3-Gi2 cells compared with in their relative controls (HEY A8-ctrli and SKOV3-ctrli) (Figure 1G-H).

To test whether GMFG functions to modulate the FAK signaling, we first examined the effects of GMFG on the FAs-associated signaling molecules in GMFG overexpression and silencing cell lines. As shown in Figure 11, compared with in control cells, the expression of FAK, p-FAK (Tyr397), Talin, p-Talin (Ser425), pPaxillin (Tyr118), Src and p-Src (Tyr416) but not Paxillin was remarkably stimulated by overexpression of GMFG, whereas the expression levels of these proteins were decreased in GMFG-silencing cell lines, suggesting that GMFG may activate the FAK-associated signal pathway. To further strengthen this point, we performed a rescue experiment by overexpression of GMFG in HEY A8-Gi-2 cells (subsequently abbreviated as 'HEY A8-Gi') to generate HEY A8-Gi-G cell line (Figure 1 J). Analysis by WB showed that FAs proteins including FAK, p-FAK (Tyr397), Talin, p-Talin (Ser425), Src and p-Src (Tyr416) were substantially upregulated in HEY A8-Gi-G cells compared with relative controls (Figure 1K). These data further proved that GMFG may directly activate the FAK signal pathway. 


\section{GMFG mediates the ECM1-induced activation of the FAK signaling through direct binding to the phosphorylated FAK and Talin}

To determine whether GMFG mediates the ECM1-induced activation of FAK signaling, we overexpressed GMFG in HEY A8-Ei cells to establish HEY A8-Ei-G cell line and silenced GMFG in HEY A8-Ei-A cells to generate HEY A8-Ei-A-Gi cell line, which were verified by WB (Figure 2A). Further tests showed that delivery of GMFG cDNA into HEY A8-Ei cells obviously increased the expression levels of FAK, p-FAK (Tyr397), Talin, p-Talin (Ser425), p-Paxillin (Tyr118) and p-Src (Tyr416), while silencing of GMFG in HEY A8-Ei-A cells markedly decreased the expression of these proteins (Figure 2B), indicating that GMFG is a downstream molecule of ECM1 and mediates the ECM1-induced ovarian tumorigenesis through the activation of FAK/Talin/Paxillin/Src signaling molecules.

To further understand how GMFG activates the FAK signaling, we tested whether GMFG physically binds to FAs-associated proteins by co-IP assays. As shown in Figure 2C, GMFG bound to the phosphorylated FAK (Tyr397) and Talin (Ser425), but not to the non-phosphorylated proteins, while the binding of GMFG to other FA proteins including Paxillin, p-Paxillin (Tyr118), Src, and p-Src (Tyr416) was not detected. The co-localization of GMFG with p-FAK (Tyr397) and p-Talin (Ser425) was also detected by IF staining (Figure 2D-E). These data indicate that GMFG activates the FAK signal pathway potentially through direct binding to p-FAK (Tyr397) and p-Talin (Ser425).

\section{GMFG promotes the proliferation, migration and invasion of ovarian cancer cells}

As GMFG and FA dynamics are vital for cell division, we first conducted a series of in vitro experiments to test the cell proliferation and tumorigenic capabilities of cell lines with different levels of GMFG. We found that the cell growth, the number of cell clones and colonies were promoted by GMFG overexpression, but were inhibited by GMFG silencing as tested by CCK8 assay (Figure 3A-B), plate cloning formation assay (Figure $3 \mathrm{C}-\mathrm{D}$ ) and anchorage-independent assays (Figure 3E-F). Considering GMFG and FAs dynamics are also important to cell migration and invasion, we then measured the migration and invasion abilities of cell lines with different expression levels of GMFG by using wound healing assay, migration and invasion experiments. The results showed that overexpression of GMFG enhanced cell wound healing (Figure 3G-H), transwell migration (Figure 3l-J), and transwell invasion

(Figure 3K-L), indicating that GMFG enhances the migration and invasion of ovarian cancer cells.

\section{FAK inhibition suppresses GMFG-induced ovarian cancer cell proliferation and migration}

To prove that GMFG accelerates the proliferation and migration of ovarian cancer through the FAKdependent manner, HEY-G cells were subjected to the FAK inhibitors Defactinib, GSK2256098 or PF573228 to suppress the phosphorylation of FAK at Tyr397. WB analysis demonstrated that the expression of p-FAK (Tyr397), p-Talin (Ser425), and p-Src (Tyr416) was remarkably decreased in inhibitortreated HEY-G cells in comparison with that of these proteins in DMSO-treated HEY-G cells and blank HEY-G cells, showing that the FAK signal molecules were blocked by these inhibitors (Figure 4A). Further tests showed that, treatment of cells with Defactinib significantly inhibited cell proliferation and migration 
as shown by CCK8 assay (Figure 4B), cloning formation assay (Figure 4C-D), and wound healing assay (Figure 4E-F). These results indicate that the enhanced proliferation and migration by GMFG overexpression can be sufficiently reversed by the chemical compound inhibiting the FAK phosphorylation at Tyr397. Thus, GMFG promotes the proliferation and migration of ovarian cancer potentially through functioning in the FAK signaling pathway.

\section{GMFG determines the chemoresistance, tumor growth, metastasis, and prognosis of ovarian cancer}

Because cytoskeleton organization is associated with paclitaxel resistance, we investigated the impact of GMFG on cell sensitivity to paclitaxel by cell viability assay. As shown in Figure 5A-B, cells overexpressing GMFG appeared more resistant to paclitaxel with a significantly higher IC50 value than cells expressing vector. Consistently, GMFG knockdown cells were relatively sensitive to paclitaxel with a lower IC50 value when compared with control cells (Figure 5C-D). Furthermore, inhibition of FAK phosphorylation at Tyr397 promoted the sensitivity of HEY-G cells to paclitaxel treatment with a reduced IC50 value, compared with relative controls (Figure 5E-F). These results suggest that GMFG facilitates the paclitaxel resistance phenotype of ovarian cancer cells most likely through the FAK pathway-induced FAs dynamics.

Next, we carried out animal assays to explore whether GMFG could promote in vivo xenograft tumor growth and intraperitoneal metastasis. As shown in Figure 5G, by intraperitoneal inoculation of cancer cells, the weight of tumor nodules derived from animals injected with HEY-G cells was significantly increased compared with that from animals applied with HEY-V cells, implying that GMFG promotes the tumor growth and intraperitoneal metastasis of ovarian cancer. To extend our study to patients' level, we investigate the prognostic significance of GMFG in ovarian cancer patients. A total of 1,415 and 1,656 patients were collected from TCGA and GEO database to conduct progression-free survival (PFS) and overall survival (OS) analysis in Kaplan-Meier Plotter website respectively. We found that high expression of GMFG is associated with a poor PFS $(\mathrm{HR}=1.42,95 \% \mathrm{Cl}: 1.1-1.82, \mathrm{P}=0.0065$, Figure $5 \mathrm{H})$, but not with a poorer OS (HR $=0.83,95 \% \mathrm{Cl}: 0.66-1.05, \mathrm{P}=0.11$, Figure $5 \mathrm{I})$.

Additionally, IHC were conducted to assess the association between the expressions of GMFG and FAKpathway proteins in the murine tumor tissues of this study. As indicated by the representative staining images in Figure 6, the expressions of GMFG, FAK, p-FAK (Tyr397), Talin, p-Talin (Ser425), Src, p-Src (Tyr416) and p-Paxillin (Tyr118) were higher in HEY-G-derived tumors than those in HEY-V-derived tumors, while the expressions of Paxillin were similar in the two groups. These data were consistent with the protein expression level detected in human ovarian cancer cell lines HEY-G and HEY-V using WB analysis. Besides, we also assessed the expression of proliferating cell nuclear antigen (PCNA), a well-known marker that is associated with high cell proliferation rate ${ }^{19}$. Higher PCNA expression was also detected in HEY-G-derived tumor tissue in relative to HEY-V-derived tumors, indicating a higher proliferation rate in the former tumor tissue. The IHC results imply a positive relationship between GMFG and FAK pathwayassociated proteins in tumor tissues. To be specific, the expression of GMFG and FAK pathwayassociated proteins were increased simultaneously in more aggressive tumor tissues, this emphasizes the vital role of GMFG in promoting FAK signaling pathway. 


\section{Discussion}

Increasing studies have demonstrated that GMFG modulates actin dynamics vital for multiple biological processes, such as angiogenesis and cell migratory behavior ${ }^{20,21}$. However, the impact of GMFG on the tumorigenicity remains largely unknown. According to literature, Zuo and colleagues reported that overexpression of GMFG promotes the migration and invasion of ovarian cancer cells, and that high expression of GMFG is associated with advanced FIGO stages, chemoresistance and poor prognosis of the disease ${ }^{22}$. Wang et al. showed that the migration and invasion of colorectal cancer cells can be enhanced by GMFG, and higher GMFG expression in colorectal cancer patients are linked to positive lymph node metastasis status ${ }^{23}$. Moreover, they further found that knockdown of GMFG in colorectal cancer cells decreases the expression of MMP2 and Vimentin, but increases the expression of E-cadherin 23. These intriguing studies initiated the investigation on the role of GMFG in cancers, but further study of the underlying pathway and in vivo experiment are needed to identify the pro-tumor function of GMFG.

In our study, we explored the function and the underlying mechanism of GMFG using in vitro and in vivo experiments, and studied GMFG as a downstream molecule of ECM1 in ovarian cancer. We found that GMFG enhances ovarian cancer cell proliferation, colony formation, migration, invasion, and paclitaxel resistance in vitro, and stimulates ovarian cancer growth and intraperitoneal metastasis in vivo. The mechanistic study indicated that the binding of GMFG to p-FAK (Tyr397) and p-Talin (Ser425) might be the key point to activate the FAK signaling pathway, thus leading to ovarian cancer progression. Besides, GMFG is a downstream molecule of ECM1 and it mediates the ECM1-induced FAK signaling. In addition, GMFG overexpression is associated with poor PFS for ovarian cancer patients. Our study provides an insightful view to understand the function and the underlying mechanism of GMFG in ovarian tumorigenesis.

Both FAK and ADFs promote FAs turnover to reduce FAs formation, leading to the elevated cell motility and the decreased cell adhesion 24,25 . The dynamics of FAs and cytoskeleton are closely related to each other, and GMFG is reported to enhance the migration of immune cells $s^{6,26}$ and airway smooth muscle cell 27 through FAs turnover. The interaction between the activated FAK and cytoskeletons for actin remodeling is also reported in many cancer cells. For instance, in pancreatic ductal adenocarcinoma, compared with radiosensitive cells, a radio-resistant cell line with a strong migration and high protein phosphorylation is closely associated with cytoskeleton dynamics including FAK activation and actin remodeling ${ }^{28}$. In ovarian cancer cells, the cytoskeletal Rho signaling pathway enhances the autophosphorylation of FAK induced by Lysophosphatidic acid (LPA) to promote the LPA-stimulated cell migratory ability ${ }^{29}$. On the contrary, the impairment of cytoskeletal reorganization and the inactivation of c-Src/FAK can jointly suppress the growth of breast cancer ${ }^{30}$. Jin et al. reported that inhibition of FAK pathway re-sensitizes the drug-resistant cancer cells to paclitaxel ${ }^{31}$. Xu and colleagues demonstrated that impairment of FAK phosphorylation at Tyr397 inhibits breast cancer cell metastasis ${ }^{32}$. Consistent with these studies, our study also found a positive interaction between GMFG and FAK signaling 
activation through which to enhance the growth, metastasis and paclitaxel resistance of ovarian cancer cells.

In addition to the phosphorylation of FAK at Tyr397 site, Talin phosphorylation at Ser425, Paxillin phosphorylation at Tyr118, and Src phosphorylation at Tyr416 are also very essential to the activation of FAK signaling pathway ${ }^{33,34}$. Jin et al. reported that Talin Ser 425 phosphorylation, but not total Talin, was associated with prostate cancer bone metastasis ${ }^{35}$. The phosphorylation of Paxillin at Tyr118 by Src increased Bcl-2 expression and contributed to cancer chemoresistance ${ }^{36,37}$. The study of Kim et al. found that the phosphorylation of FAK at Tyr397 together with the phosphorylation of Src at Tyr416 drive epithelial-to-mesenchymal transition in cervical cancer ${ }^{38}$. Consistently, according to our findings, GMFG can enhance the expressions of these phosphorylated proteins to activate FAK pathway, thereby promoting the progression of ovarian cancer.

To conclude, our study suggests that GMFG may activate the FAK/Talin/Paxillin/Src signaling potentially via direct binding to p-FAK (tyr397) and p-Talin (ser425) to mediate the ECM1-induced ovarian tumorigenesis, and that GMFG promotes the tumorigenesis and chemoresistance of ovarian cancer most likely through the FAK pathway-induced FAs dynamics. Besides, the high expression of GMFG was associated with poor prognosis, lymph node metastasis and venous invasion for ovarian cancer patients. Thus, targeting the oncogenic ECM1-GMFG-FAK axis may be a promising therapeutic strategy for ovarian cancer patients.

\section{Abbreviations}

ADF-H, actin-depolymerizing factor homology; Arps, actin-related proteins; ATCC, American Tissue Culture Collection; ECM, extracellular matrix; ECM1, extracellular matrix protein 1; FAK, focal adhesion kinase; FAs, focal adhesions; FIGO, International Federation of Gynecology and Obstetrics; GEO, Gene Expression Omnibus; GMFG, glia maturation factor- $\gamma$; LPA, lysophosphatidic acid; MMP2, matrix metalloproteinase 2; OS, overall survival; PCNA, proliferating cell nuclear antigen; PFS, progression-free survival; PTK, protein tyrosine kinase; TCGA, The Cancer Genome Atlas.

\section{Declarations}

\section{Author contributions}

GY and CR designed and directed the study, AL and CL conducted experiments, analyzed data and wrote the manuscript, JW, LY, RD, HL and YY helped to conduct several experiments. All authors read and approved the final manuscript.

\section{Data availability statement}

All data generated or analyzed during this study are included in this published article [and its supplementary information files]. 


\section{Ethics approval}

The animal assay was approved by the Institutional Animal Care and Use Committee of East China Normal University (reference number: m20200801), and was carried out in accordance with the ARRIVE guidelines (https://arriveguidelines.org).

\section{Consent for publication}

Not applicable.

\section{Funding}

This study was supported by the National Natural Science Foundation of China (No. 81772789, 81572553, and 81372797), and Natural Science Research Foundation of Minhang District, Shanghai (No.2019MHZ041).

\section{Acknowledgement}

The authors would like to acknowledge the mice that were sacrificed for this study.

\section{Competing interests}

The authors declare that they have no competing interests.

\section{References}

1. Torre LA, Trabert B, DeSantis CE, Miller KD, Samimi G, Runowicz CD, Gaudet MM, Jemal A, Siegel RL. Ovarian cancer statistics, 2018. CA Cancer J Clin 2018;68:284-96.

2. Peres LC, Cushing-Haugen KL, Kobel M, Harris HR, Berchuck A, Rossing MA, Schildkraut JM, Doherty JA. Invasive Epithelial Ovarian Cancer Survival by Histotype and Disease Stage. Journal of the National Cancer Institute 2019;111:60-68.

3. Lan A, Yang G. Clinicopathological parameters and survival of invasive epithelial ovarian cancer by histotype and disease stage. Future Oncol 2019;15:2029-39.

4. Yin H, Wang J, Li H, Yu Y, Wang X, Lu L, Lv C, Chang B, Jin W, Guo W, Ren C, Yang G. Extracellular matrix protein-1 secretory isoform promotes ovarian cancer through increasing alternative mRNA splicing and stemness. Nature communications 2021;12:4230.

5. Lan A, Ren C, Wang X, Tong G, Yang G. Bioinformatics and survival analysis of glia maturation factor- $\gamma$ in pan-cancers. BMC Cancer 2021;21:423.

6. Lippert DN, Wilkins JA. Glia maturation factor gamma regulates the migration and adherence of human T lymphocytes. BMC Immunol 2012;13:21.

7. Aerbajinai W, Liu L, Chin K, Zhu J, Parent CA, Rodgers GP. Glia maturation factor-y mediates neutrophil chemotaxis. J Leukoc Biol 2011;90:529-38. 
8. Schimmel L, Fukuhara D, Richards M, Jin Y, Essebier P, Frampton E, Hedlund M, Dejana E, ClaessonWelsh L, Gordon E. c-Src controls stability of sprouting blood vessels in the developing retina independently of cell-cell adhesion through focal adhesion assembly. Development 2020;147.

9. Young LE, Higgs HN. Focal Adhesions Undergo Longitudinal Splitting into Fixed-Width Units. Curr Biol 2018;28:2033-45.e5.

10. Brami-Cherrier K, Gervasi N, Arsenieva D, Walkiewicz K, Boutterin MC, Ortega A, Leonard PG, Seantier B, Gasmi L, Bouceba T, Kadare G, Girault JA, et al. FAK dimerization controls its kinase-dependent functions at focal adhesions. The EMBO journal 2014;33:356-70.

11. Fan T, Chen J, Zhang L, Gao P, Hui Y, Xu P, Zhang X, Liu H. Bit1 knockdown contributes to growth suppression as well as the decreases of migration and invasion abilities in esophageal squamous cell carcinoma via suppressing FAK-paxillin pathway. Molecular cancer 2016;15:23.

12. McGrail DJ, Khambhati NN, Qi MX, Patel KS, Ravikumar N, Brandenburg CP, Dawson MR. Alterations in ovarian cancer cell adhesion drive taxol resistance by increasing microtubule dynamics in a FAKdependent manner. Sci Rep 2015;5:9529.

13. Balsas P, Palomero J, Eguileor Á, Rodríguez ML, Vegliante MC, Planas-Rigol E, Sureda-Gómez M, Cid MC, Campo E, Amador V. SOX11 promotes tumor protective microenvironment interactions through CXCR4 and FAK regulation in mantle cell lymphoma. Blood 2017;130:501-13.

14. Nader GP, Ezratty EJ, Gundersen GG. FAK, talin and PIPKIy regulate endocytosed integrin activation to polarize focal adhesion assembly. Nat Cell Biol 2016;18:491-503.

15. Yang G, Xiao X, Rosen DG, Cheng X, Wu X, Chang B, Liu G, Xue F, Mercado-Uribe I, Chiao P, Du X, Liu J. The biphasic role of NF-kappaB in progression and chemoresistance of ovarian cancer. Clinical cancer research : an official journal of the American Association for Cancer Research 2011;17:218194.

16. Yang G, Rosen DG, Mercado-Uribe I, Colacino JA, Mills GB, Bast RC, Jr., Zhou C, Liu J. Knockdown of p53 combined with expression of the catalytic subunit of telomerase is sufficient to immortalize primary human ovarian surface epithelial cells. Carcinogenesis 2007;28:174-82.

17. Yang G, Rosen DG, Zhang Z, Bast RC, Jr., Mills GB, Colacino JA, Mercado-Uribe I, Liu J. The chemokine growth-regulated oncogene 1 (Gro-1) links RAS signaling to the senescence of stromal fibroblasts and ovarian tumorigenesis. Proc Natl Acad Sci U S A 2006;103:16472-7.

18. Gyorffy B, Lánczky A, Szállási Z. Implementing an online tool for genome-wide validation of survivalassociated biomarkers in ovarian-cancer using microarray data from 1287 patients. Endocr Relat Cancer 2012;19:197-208.

19. Lv Q, Zhang J, Yi Y, Huang Y, Wang Y, Wang Y, Zhang W. Proliferating Cell Nuclear Antigen Has an Association with Prognosis and Risks Factors of Cancer Patients: a Systematic Review. Mol Neurobiol 2016;53:6209-17.

20. Ikeda K, Kundu RK, Ikeda S, Kobara M, Matsubara H, Quertermous T. Glia maturation factor-gamma is preferentially expressed in microvascular endothelial and inflammatory cells and modulates actin cytoskeleton reorganization. Circ Res 2006;99:424-33. 
21. Zuo P, Fu Z, Tao T, Ye F, Chen L, Wang X, Lu W, Xie X. The expression of glia maturation factors and the effect of glia maturation factor- $\gamma$ on angiogenic sprouting in zebrafish. Exp Cell Res 2013;319:707-17.

22. Zuo P, Ma Y, Huang Y, Ye F, Wang P, Wang X, Zhou C, Lu W, Kong B, Xie X. High GMFG expression correlates with poor prognosis and promotes cell migration and invasion in epithelial ovarian cancer. Gynecol Oncol 2014;132:745-51.

23. Wang H, Chen Z, Chang H, Mu X, Deng W, Yuan Z, Yao F, Liu Y, Mai R, Wu B. Expression of glia maturation factor $\mathrm{Y}$ is associated with colorectal cancer metastasis and its downregulation suppresses colorectal cancer cell migration and invasion in vitro. Oncol Rep 2017;37:929-36.

24. Kanellos G, Zhou J, Patel H, Ridgway RA, Huels D, Gurniak CB, Sandilands E, Carragher NO, Sansom OJ, Witke W, Brunton VG, Frame MC. ADF and Cofilin1 Control Actin Stress Fibers, Nuclear Integrity, and Cell Survival. Cell Rep 2015;13:1949-64.

25. Garcia-Keller C, Scofield MD, Neuhofer D, Varanasi S, Reeves MT, Hughes B, Anderson E, Richie CT, Mejias-Aponte C, Pickel J, Hope BT, Harvey BK, et al. Relapse-Associated Transient Synaptic Potentiation Requires Integrin-Mediated Activation of Focal Adhesion Kinase and Cofilin in D1Expressing Neurons. J Neurosci 2020;40:8463-77.

26. Aerbajinai W, Liu L, Zhu J, Kumkhaek C, Chin K, Rodgers GP. Glia Maturation Factor-y Regulates Monocyte Migration through Modulation of $\beta 1$-Integrin. J Biol Chem 2016;291:8549-64.

27. Gerlach BD, Tubbesing K, Liao G, Rezey AC, Wang R, Barroso M, Tang DD. Phosphorylation of GMFY by c-Abl Coordinates Lamellipodial and Focal Adhesion Dynamics to Regulate Airway Smooth Muscle Cell Migration. Am J Respir Cell Mol Biol 2019;61:219-31.

28. Wiechmann S, Saupp E, Schilling D, Heinzlmeir S, Schneider G, Schmid RM, Combs SE, Kuster B, Dobiasch S. Radiosensitization by Kinase Inhibition Revealed by Phosphoproteomic Analysis of Pancreatic Cancer Cells. Mol Cell Proteomics 2020;19:1649-63.

29. Bian D, Mahanivong C, Yu J, Frisch SM, Pan ZK, Ye RD, Huang S. The G12/13-RhoA signaling pathway contributes to efficient lysophosphatidic acid-stimulated cell migration. Oncogene 2006;25:2234-44.

30. Lee KJ, Kim Y, Kim MS, Ju HM, Choi B, Lee H, Jeoung D, Moon KW, Kang D, Choi J, Yook JI, Hahn JH. CD99-PTPN12 Axis Suppresses Actin Cytoskeleton-Mediated Dimerization of Epidermal Growth Factor Receptor. Cancers (Basel) 2020;12.

31. Jin $\mathrm{H}, \mathrm{He} \mathrm{Y}$, Zhao P, Hu Y, Tao J, Chen J, Huang Y. Targeting lipid metabolism to overcome EMTassociated drug resistance via integrin $\beta 3 / F A K$ pathway and tumor-associated macrophage repolarization using legumain-activatable delivery. Theranostics 2019;9:265-78.

32. Xu Q, Zhang J, Telfer BA, Zhang H, Ali N, Chen F, Risa B, Pearson AJ, Zhang W, Finegan KG, Ucar A, Giurisato $E$, et al. The extracellular-regulated protein kinase 5 (ERK5) enhances metastatic burden in triple-negative breast cancer through focal adhesion protein kinase (FAK)-mediated regulation of cell adhesion. Oncogene 2021. 
33. Hao H, Naomoto Y, Bao X, Watanabe N, Sakurama K, Noma K, Motoki T, Tomono Y, Fukazawa T, Shirakawa Y, Yamatsuji T, Matsuoka J, et al. Focal adhesion kinase as potential target for cancer therapy (Review). Oncology reports 2009;22:973-9.

34. Parsons JT. Focal adhesion kinase: the first ten years. Journal of cell science 2003;116:1409-16.

35. Jin JK, Tien PC, Cheng CJ, Song JH, Huang C, Lin SH, Gallick GE. Talin1 phosphorylation activates $\beta 1$ integrins: a novel mechanism to promote prostate cancer bone metastasis. Oncogene 2015;34:1811-21.

36. Wu DW, Huang CC, Chang SW, Chen TH, Lee H. Bcl-2 stabilization by paxillin confers 5-fluorouracil resistance in colorectal cancer. Cell death and differentiation 2015;22:779-89.

37. Wu DW, Wu TC, Wu JY, Cheng YW, Chen YC, Lee MC, Chen CY, Lee H. Phosphorylation of paxillin confers cisplatin resistance in non-small cell lung cancer via activating ERK-mediated Bcl-2 expression. Oncogene 2014;33:4385-95.

38. Kim YM, Muthuramalingam K, Cho M. Redox Regulation of NOX Isoforms on FAK((Y397))/SRC((Y416)) Phosphorylation Driven Epithelial-to-Mesenchymal Transition in Malignant Cervical Epithelial Cells. Cells 2020;9.

\section{Figures}


(A)

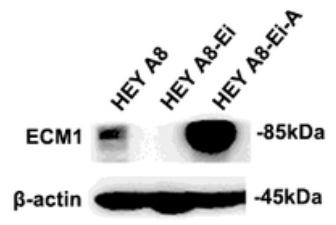

(B)

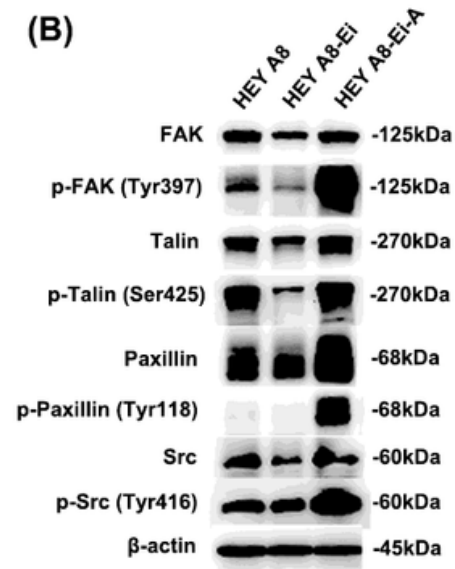

(C)

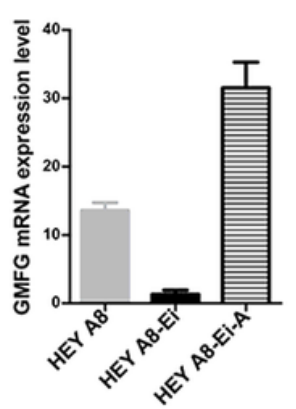

(D)

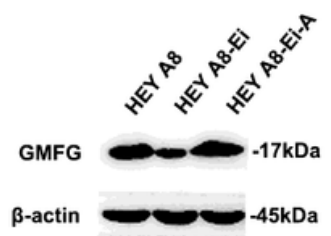

(E)

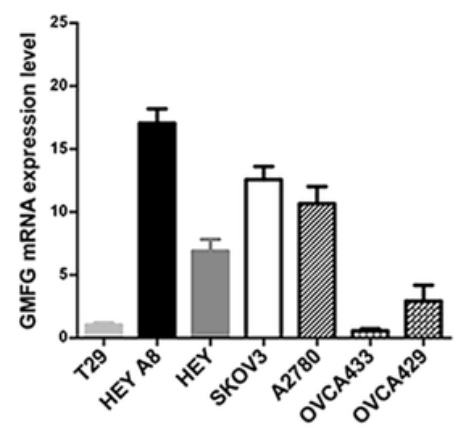

(F)

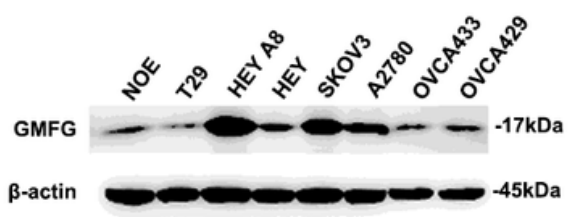

(G)

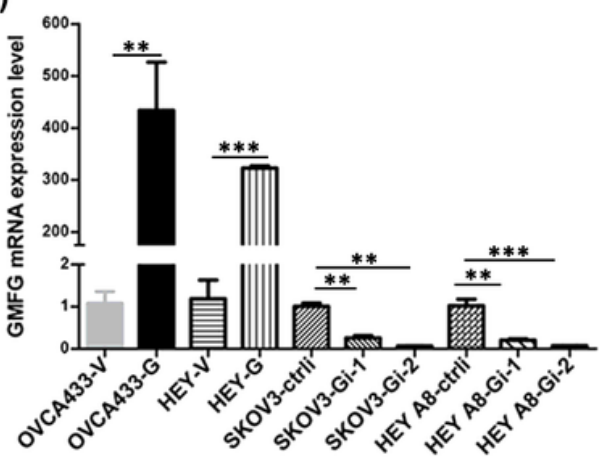

(H)

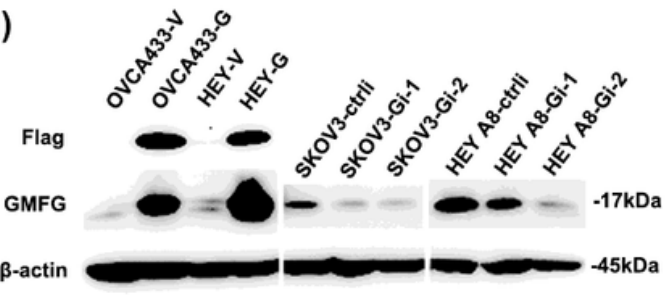

(I)

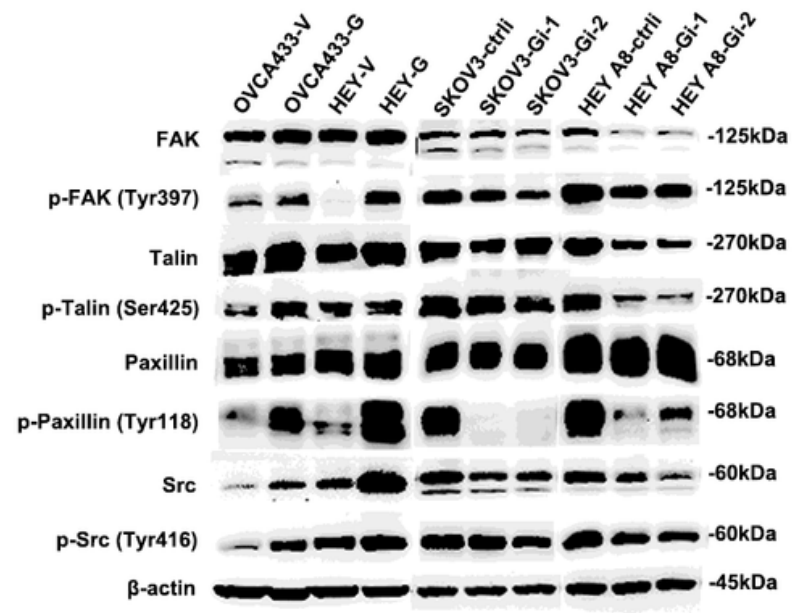

(J)

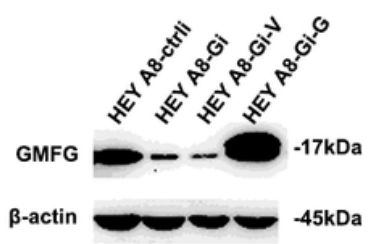

(K)

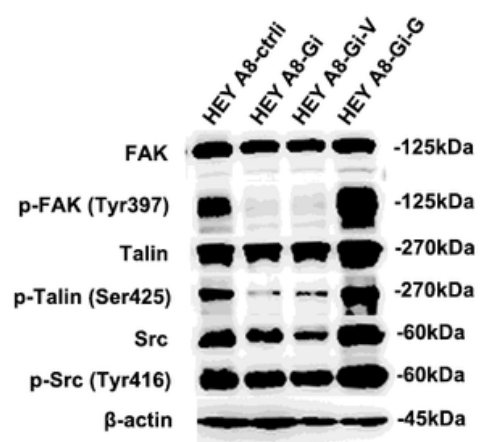

Figure 1

ECM1-indueced GMFG activates the FAK-associated signaling pathway. (A) Identification of HEY A8-Ei and HEY A8-Ei-A cells by WB. (B) Activation of the FAK signaling molecules by ECM1 tested by WB. (C) mRNA levels of GMFG in HEY A8, HEY A8-Ei, and HEY A8-Ei-A cells tested by qPCR. (D) GMFG expression in HEY A8, HEY A8-Ei, and HEY A8-Ei-A cells detected by WB. (E) mRNA levels of GMFG in epithelial ovarian cancer cell lines (HEY A8, HEY, SKOV3, A2780, OVCA433, and OVCA429), T29 and NOE tested by qPCR. (F) GMFG expression in above cell lines detected by WB. (G) Establishment of GMFG overexpression and knockdown cell lines confirmed by qPCR. (H) Establishment of GMFG overexpression and knockdown cell lines confirmed by WB. (I) Alteration of the FAK-associated signaling molecules by GMFG as tested by WB. (J) Establishment of GMFG overexpression cells in GMFG silencing cells confirmed by WB. (K) Expression of FAs proteins detected by WB in GMFG-rescued cells. 
(A)

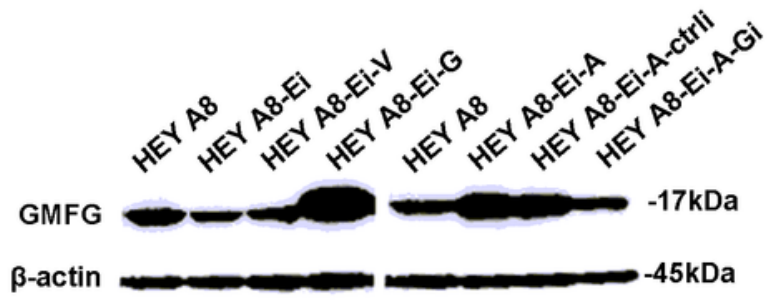

(B)

(C)

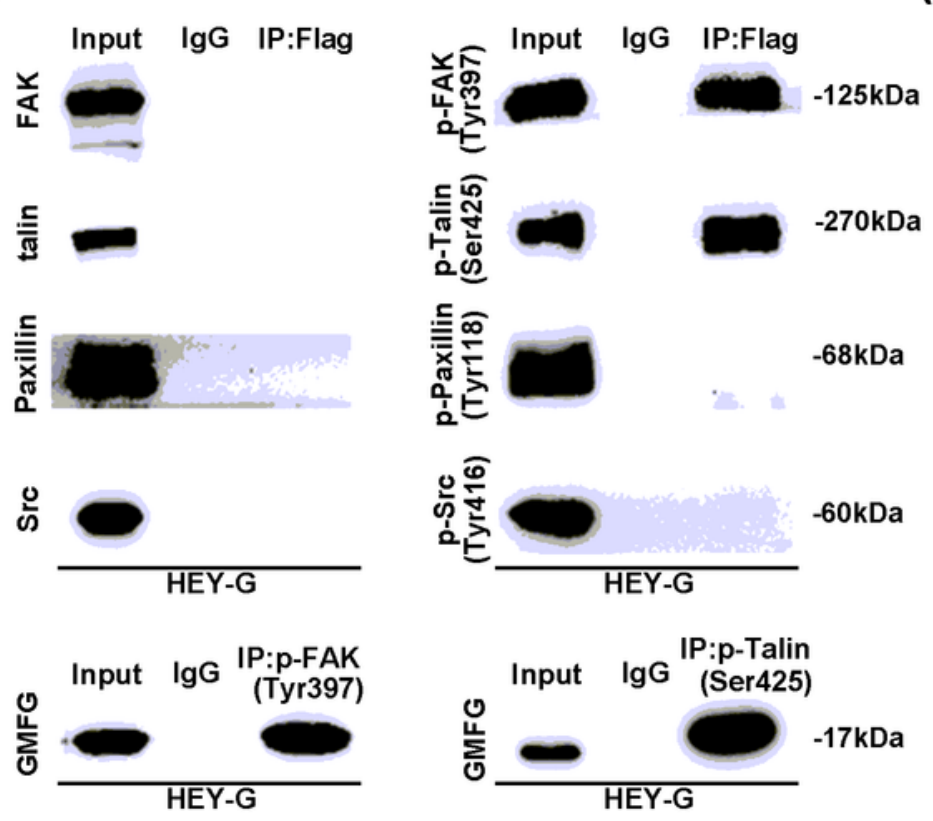

(D)

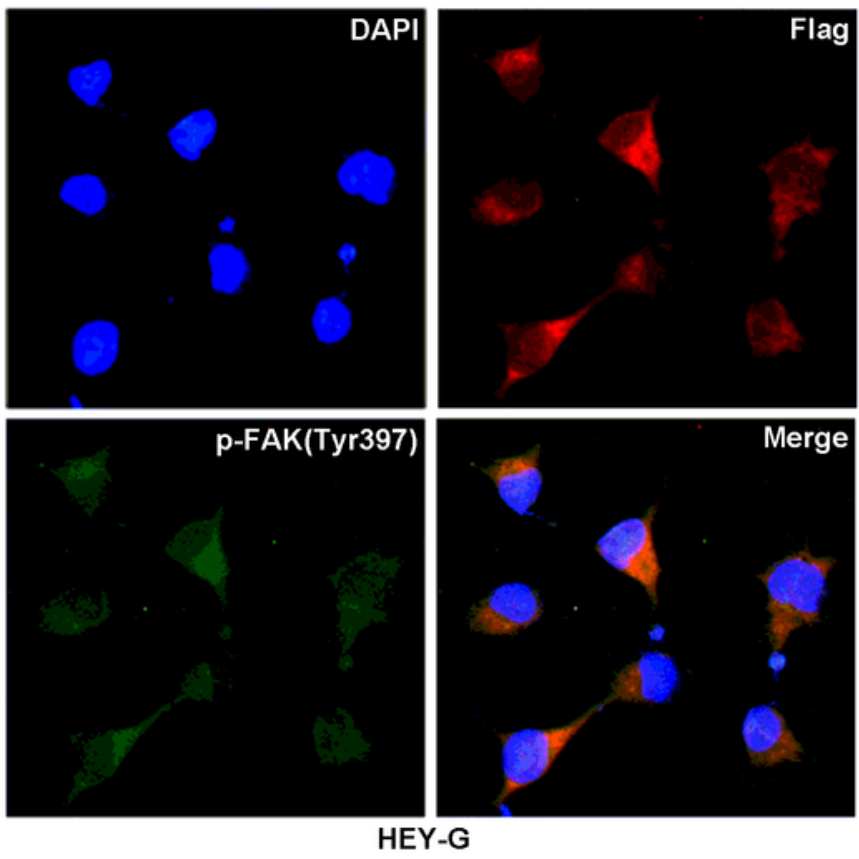

(E)

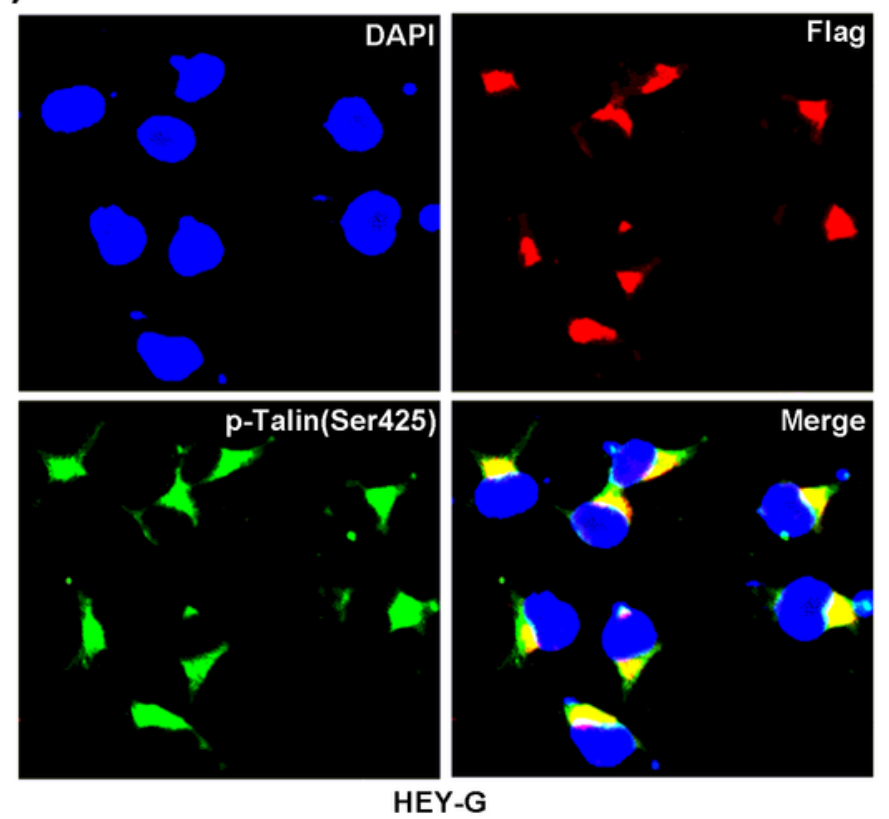

Figure 2

GMFG mediates the ECM1-induced activation of FAK signaling through direct binding to the phosphorylated FAK and Talin. (A) Overexpression or silencing of GMFG in HEY A8-Ei or HEY A8-Ei-A cell lines confirmed by WB. (B) Expression of FAs proteins detected by WB in ECM1-GMFG cells. (C) Binding of GMFG to FAs proteins detected by co-IP. (D-E) Co-localization of GMFG with p-FAK (Tyr397) (D) or pTalin (Ser425) (E) tested by IF staining. 
(A)

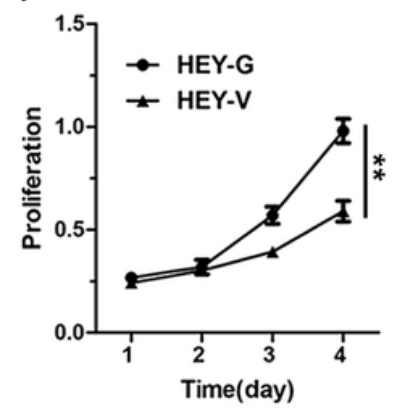

(C)

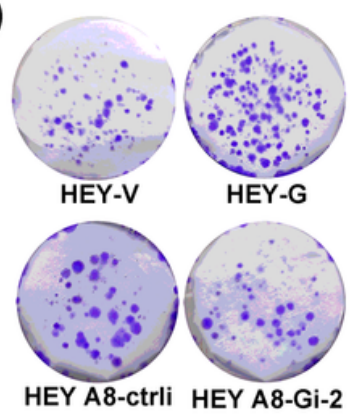

(E)

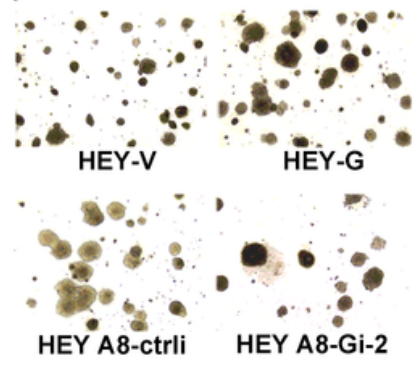

(B)

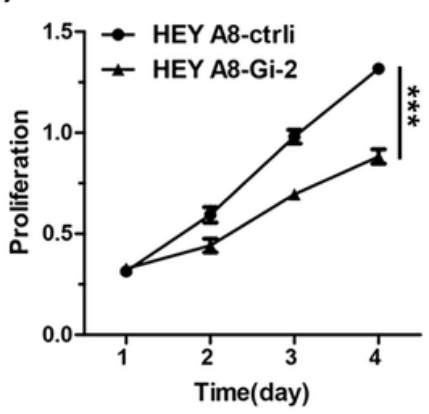

(D)

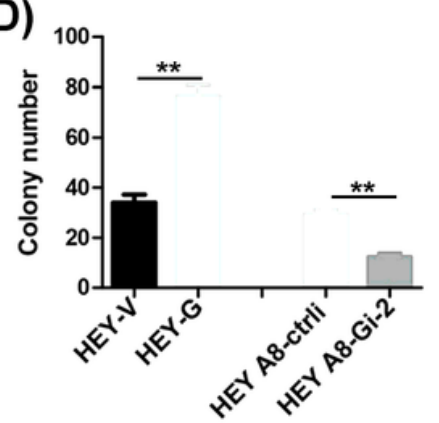

(F)

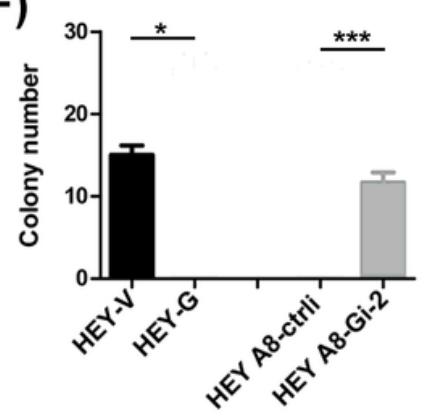

(G)

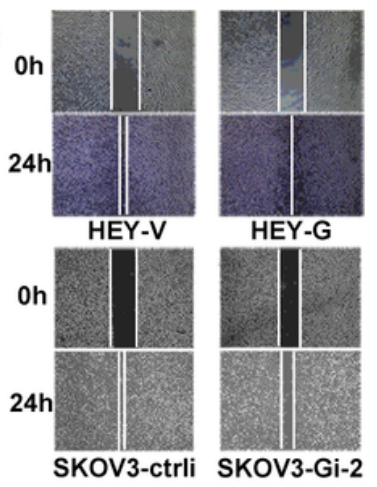

(I)

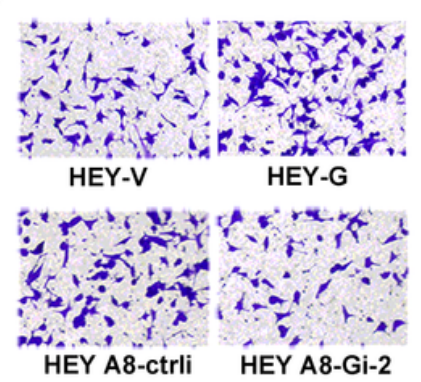

(K)

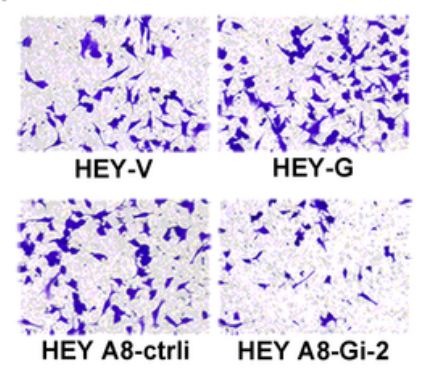

(H)

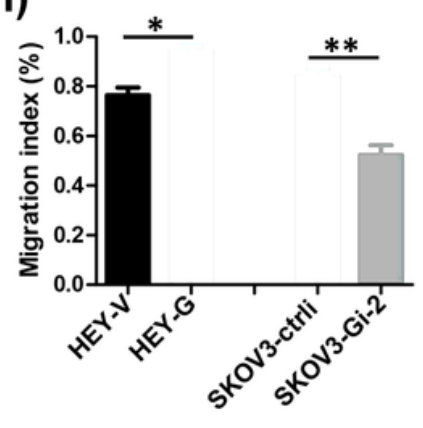

(J)

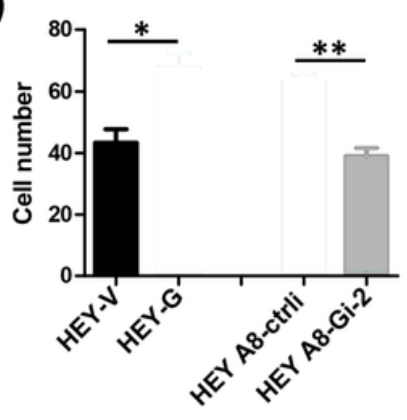

(L)

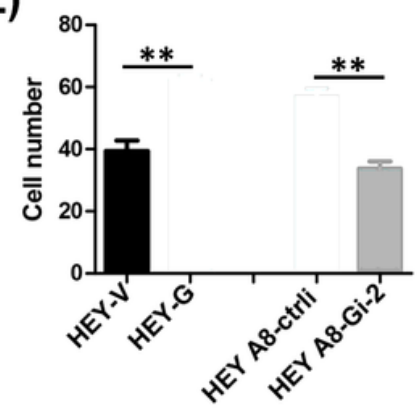

Figure 3

GMFG promotes cell proliferation, migration and invasion. (A) Detection of cell proliferation in HEY-V and HEY-G by CCK-8 at the indicated time points. (B) Detection of cell proliferation in HEY A8-ctrli and HEY A8Gi-2 by CCK-8 at the indicated time points. (C) Representative images of plate clone formation assay. (D) Quantitative analysis of plate clones. (E) Representative images of colonies from soft agar anchorageindependent growth. (F) Quantitative analysis of colonies from soft agar anchorage-independent growth. (G) Detection of cell migration by wound healing assay. $(\mathrm{H})$ Quantitative analysis of migration index from wound healing assay. (I) Detection of cell migration by transwell migration assay. (J) Quantitative analysis of migrated cells. (K) Detection of cell invasion by transwell invasion assay. (L) Quantitative analysis of invaded cells. 
(A)

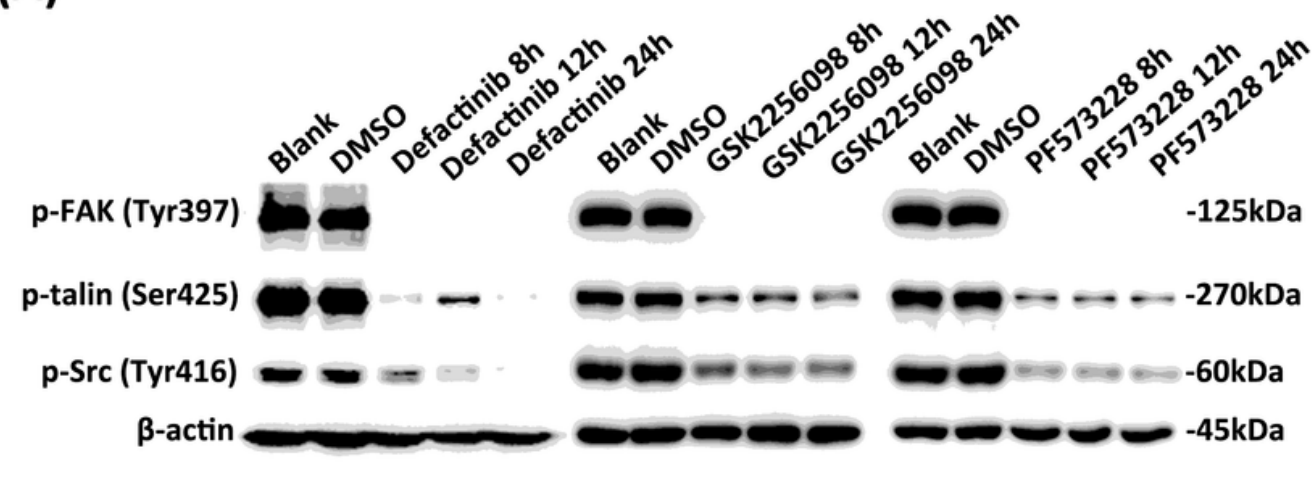

(C)
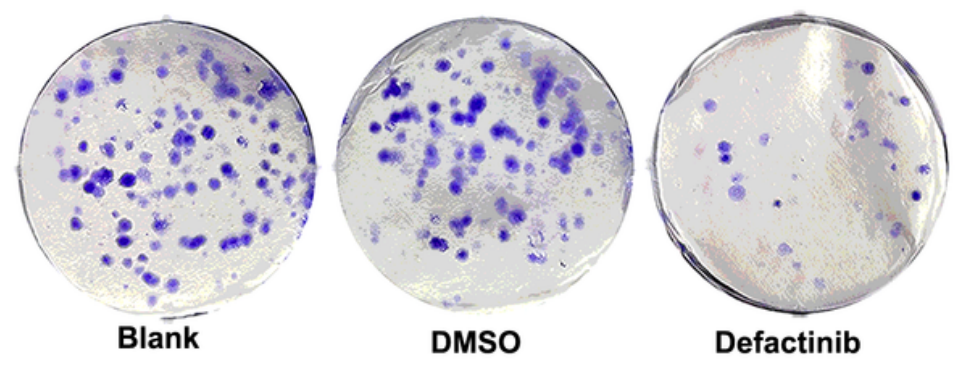

(E)

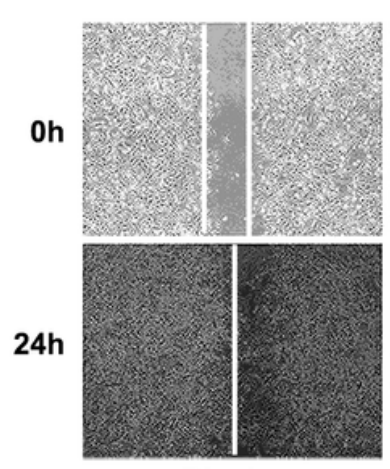

Blank

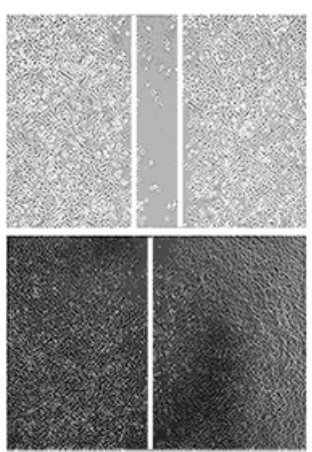

DMSO

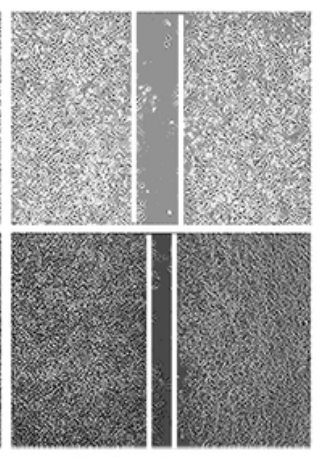

Defactinib
(B)

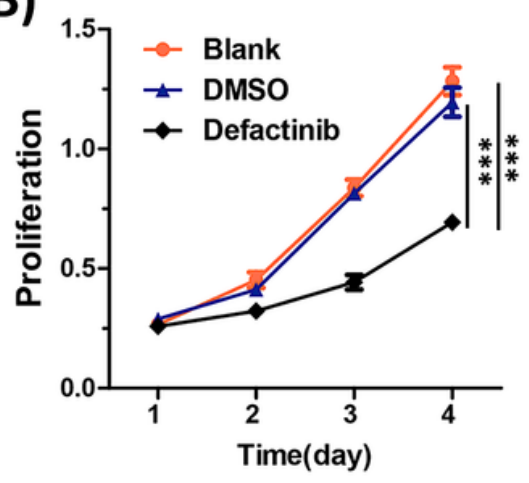

(D)

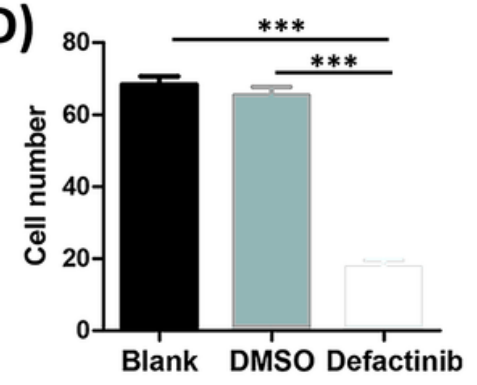

(F)

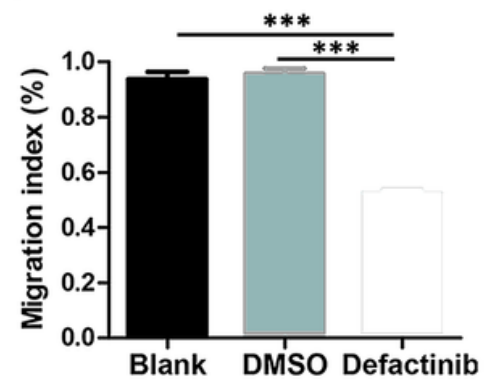

Figure 4

FAK inhibition suppresses GMFG-induced cell proliferation and migration. (A) Phosphorylation of FA proteins detected by WB in cells with or without Defactinib treatment. (B) Cell proliferation of HEY-G cells treated with or without Defactinib detected by CCK-8 assay. (C) Clone-formation of HEY-G cells treated as in (B). (D) Quantitative analysis of plate clones. (E) Migration speed of HEY-G cells treated as in (B) tested by wound healing assay. (F) Quantitative analysis of migration index. 
(A)

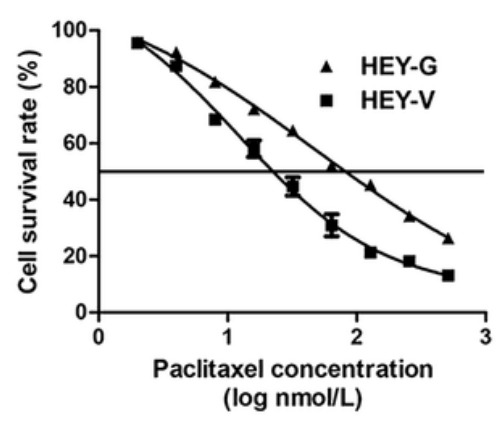

(C)

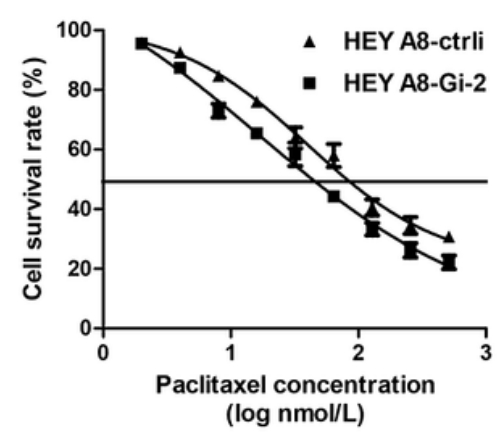

(E)

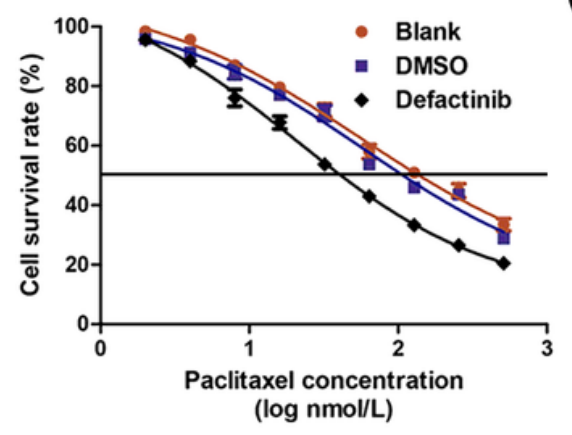

(B)

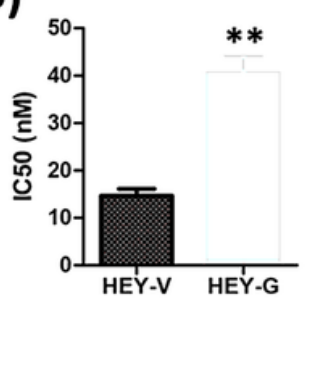

(D)

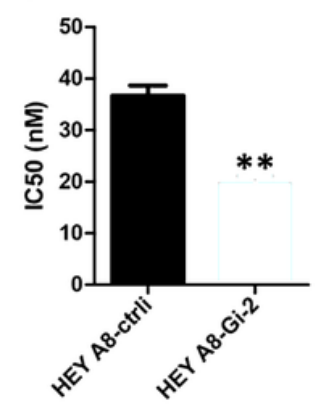

(I)

(H)

(G)
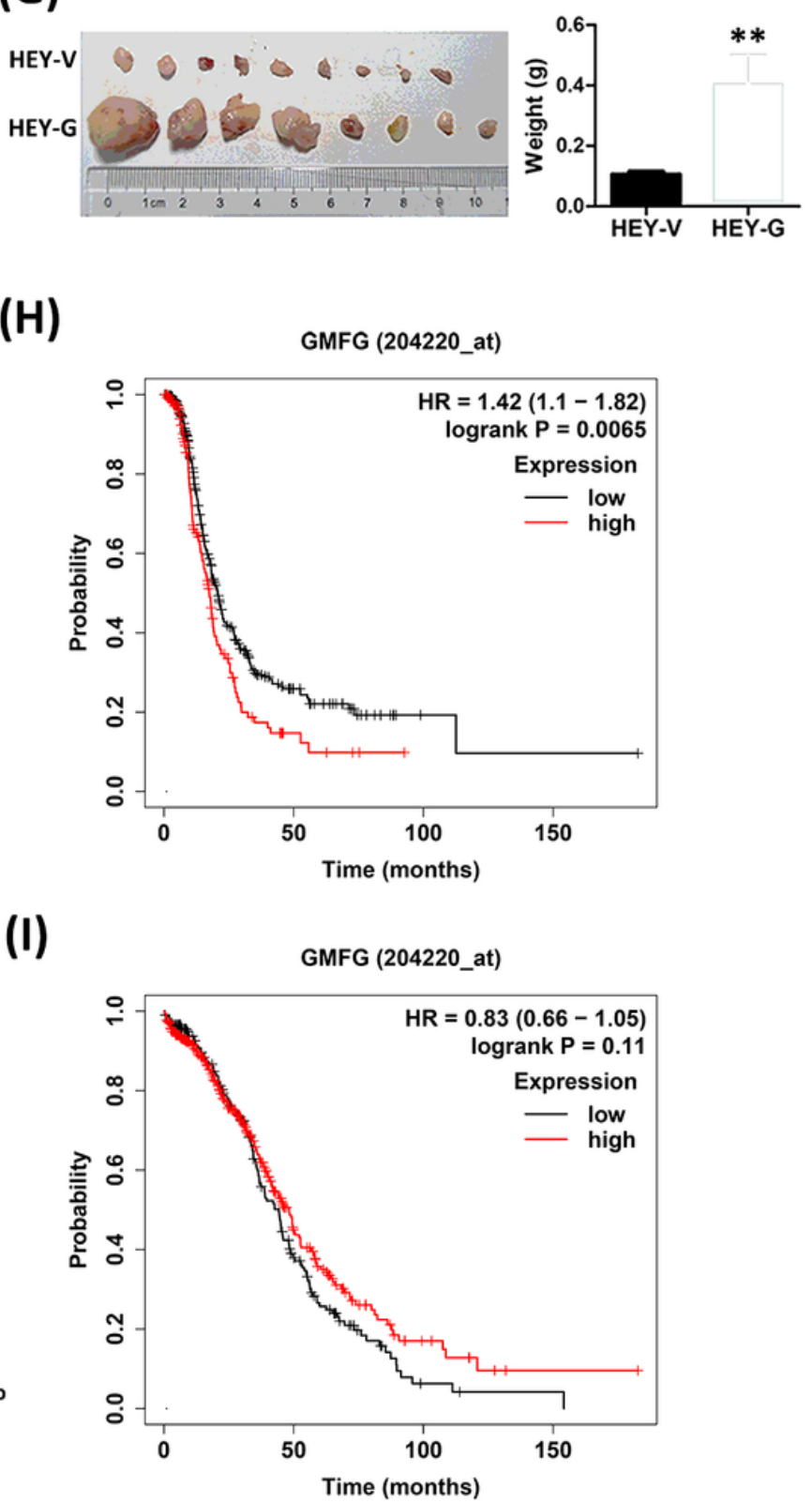

(F)

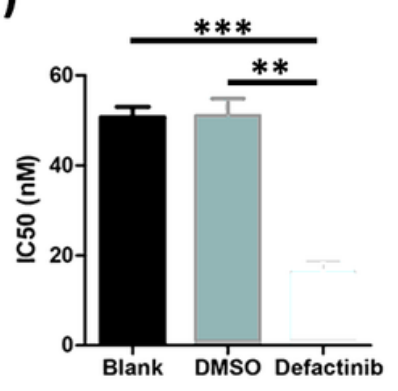

GMFG (204220_at)

$=1.42(1.1-1.82)$

Expression

- low

EY-V HEY-G

Figure 5 

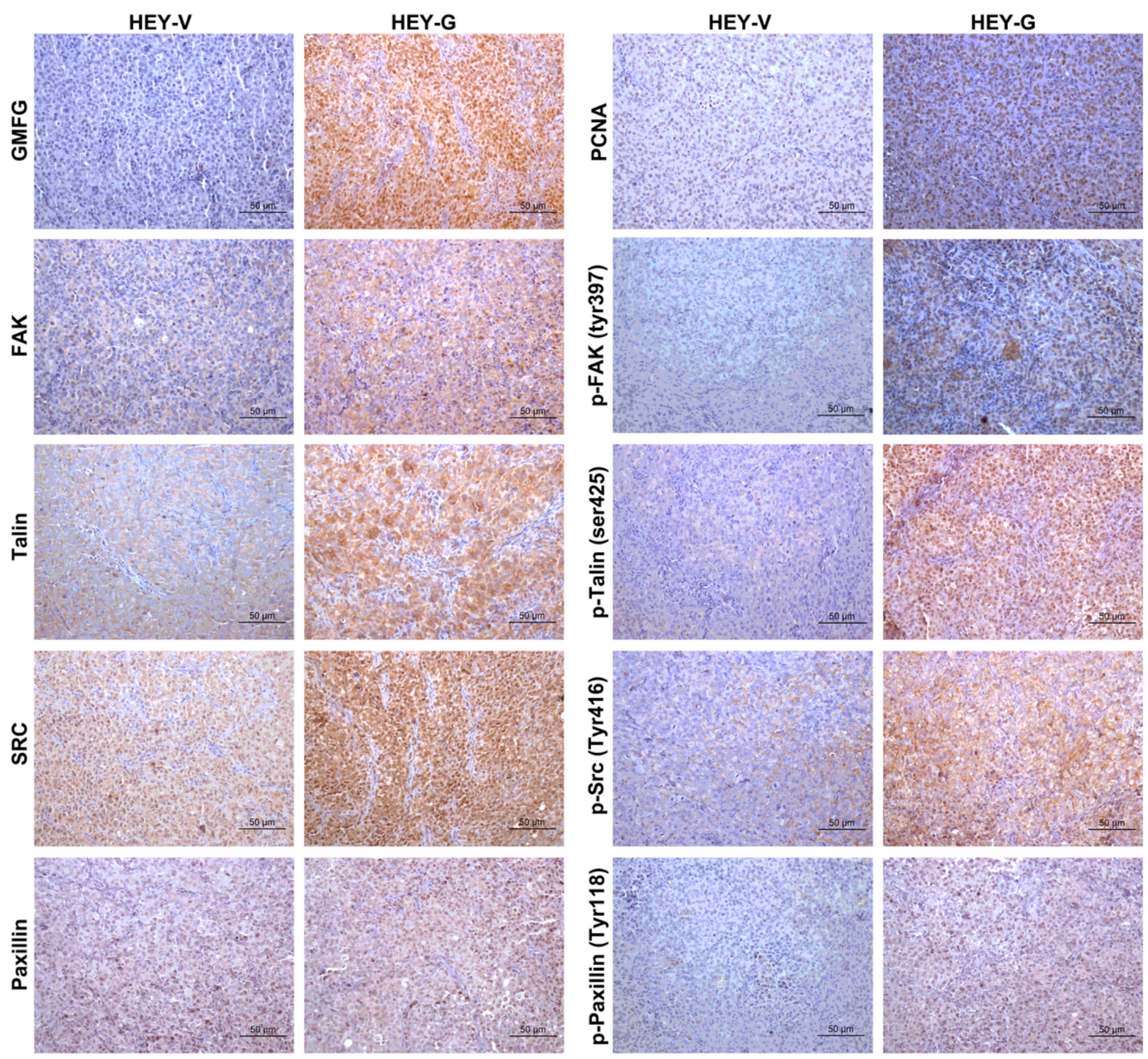

Figure 6

The expression of GMFG and FAK pathway-associated proteins were increased simultaneously in more aggressive tumor tissues. Detection of GMFG, PCNA, FAK, p-FAK (Tyr397), Talin, p-Talin (Ser425), Src, pSrc (Tyr416), Paxillin and p-Paxillin (Tyr118) expressions in xenograft tumor tissues by IHC staining (Representative images were captured at $200 \times$ magnification).

\section{Supplementary Files}

This is a list of supplementary files associated with this preprint. Click to download. 
- Supplementarylnformationfile.pdf

- Supplementarymaterials.pdf 